\title{
Research Article \\ Constructing Multi-Branches Complete Chaotic Maps That Preserve Specified Invariant Density
}

\author{
Weihong Huang \\ Nanyang Technological University, Nanyang Avenue, Singapore 639798 \\ Correspondence should be addressed to Weihong Huang, awhhuang@ntu.edu.sg
}

Received 6 February 2009; Accepted 13 October 2009

Recommended by Akio Matsumoto

A general formulation for multi-branches complete chaotic maps that preserve a specified invariant density is provided and an implication relationship among this class of maps is revealed. Such relationship helps to derive a whole family of complete chaotic maps that preserve not only the same invariant measure but also the same degree of chaos in terms of Lyapunov component.

Copyright (C 2009 Weihong Huang. This is an open access article distributed under the Creative Commons Attribution License, which permits unrestricted use, distribution, and reproduction in any medium, provided the original work is properly cited.

\section{Introduction}

Much progress has been achieved in exploring the probabilistic characteristics of nonlinear dynamics from different aspects over the last ten years. While the most general formulations of unimodal (two-branches) complete chaotic maps that preserve general form of invariant densities have been provided in [1,2], the characterization of such maps for some special forms of invariant densities are analyzed in $[2,3]$. Interests to the unimodal chaotic maps with special analytical structural forms are explored in [4-6] and the relevant statistical dynamics are investigated in [7-9]. However, most these studies are limited to complete chaotic maps with two branches only until recently in [10], where the methodologies developed in $[1,2]$ are generalized to the cases with multi-branches so that most general formulations are derived. The current research intends to continue the same endeavor through deriving more compact and more simplified formulations and to reveal an important relation among them. The results we obtain do not only provide a practical approach to construct multi-branches complete chaotic maps for arbitrary specified analytical expressible invariant densities but also help in understanding the intrinsic characteristics of such maps.

\section{Complete Chaotic Maps}

Let $J=[0,1]$ be the unitinterval. Let $F: J \rightarrow J$ be a nonsingular map. "Nonsigularity" means that $m\left(F^{-1}(X)\right)=0$ whenever $m(X)=0$ for a measurable set $X \subset J$, where $m$ denotes 
the Lebesgue measure (all mathematical functions in this paper are assumed to be piecewise continuous and differentiable).

Definition 2.1. A nonsingular map $F: J \rightarrow J$ is said to be complete if

(i) there exists a partition $A_{F}=\left\{a_{i}\right\}_{i=0}^{r}$, with $0=a_{0}<a_{1}<\cdots<a_{r-1}<a_{r}=1$, of the unit interval $J$ such that for each integer $i(i=1,2, \ldots, r)$, the restriction $F_{i}$ of $F$ to the open interval $\left(a_{i-1}, a_{i}\right)$ is a monotonically continuous function that can be extended to the closed interval $J_{i}=\left[a_{i-1}, a_{i}\right]$;

(ii) $F_{i}\left(a_{i-1}\right) \in\{0,1\}, F_{i}\left(a_{i}\right) \in\{0,1\}$, and $F_{i}\left(a_{i-1}\right)+F_{i}\left(a_{i}\right)=1$, for $i=1,2, \ldots, r$.

We will call $F_{i}$ extended to $J_{i}$ as $i$ th branch of $F, s_{i} \dot{=} F_{i}\left(a_{i}\right)-F_{i}\left(a_{i-1}\right)$ as its sign index to indicate the slopping direction of $F$ on $i$ th subinterval $J_{i}, i=1, \ldots, r$, and $S=\left\{s_{i}\right\}_{i=1}^{r}$ as the sign pattern of $F=\left\{F_{i}\right\}_{i=1}^{r}$. In particular, we will let $I_{+} \dot{\doteq}\left\{i \mid s_{i}>0\right\}$ and $I_{-} \dot{\doteq}\left\{i \mid s_{i}<0\right\}$ be the positive and negative index sets that indicate the set of branches with positive and negative slopes, respectively. For instance, the chaotic map defined by

$$
F(x)= \begin{cases}F_{1}(x)=\sqrt{3 x}, & x \in\left[0, \frac{1}{3}\right), \\ F_{2}(x)=\sqrt{\frac{4}{3}-3 x^{2}} & x \in\left[\frac{1}{3}, \frac{2}{3}\right), \\ F_{3}(x)=\sqrt{\frac{9 x^{2}-4}{5},} & x \in\left[\frac{2}{3}, 1\right]\end{cases}
$$

is complete with $A_{F}=\{0,1 / 3,2 / 3,1\}, S=\{1,-1,1\}, I_{+}=\{1,3\}$, and $I_{-}=\{2\}$.

Definition 2.2. A complete map $F$ is said to be complete chaotic (fully chaotic) if it is

(i) ergodic with respect to the Lebesgue measure,

(ii) chaotic in the probabilistic sense, that is, an absolutely continuous invariant density $\varphi$ is preserved. In other words, for

$$
\mu(x)=\int_{0}^{x} \varphi(x) d x, \quad x \in J .
$$

We have

$$
\mu(X)=\mu\left(F^{-1}(X)\right)
$$

for any set of $X \subset J$ with $m(X) \neq 0$, where $m$ denotes the Lebesgue measure.

As illustrated in Figure 1, for a complete chaotic map $F$ defined on $J=\bigcup_{i=1}^{r} J_{i}$, for any $x \in J, F$ has exact $r$ preimages, denoted as $F_{i}^{-1}(x), i=1,2, \ldots, r$. Therefore, for any $x \in J_{1}$, $F_{1}(x)$ has $r$ preimages in each subinterval $J_{i}, i \geq 1$, which are linked with $x$ through the branching functions $\omega_{i}: J_{1} \rightarrow J_{i}$, defined by

$$
\omega_{i}(x) \doteq F_{i}^{-1}\left(F_{1}(x)\right), \quad \forall x \in J_{1}, i=1,2, \ldots, r .
$$




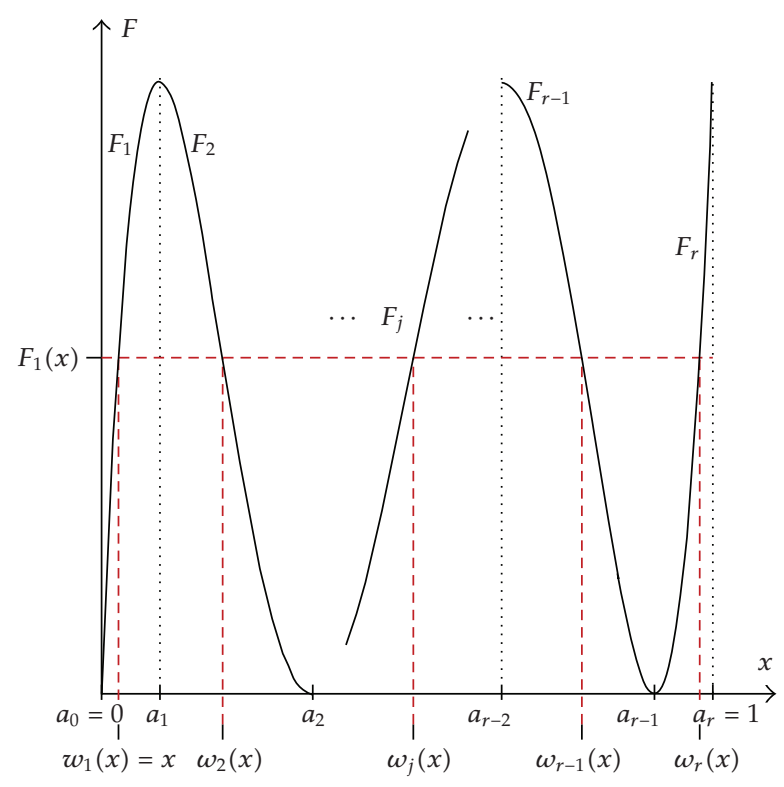

Figure 1: Illustration of branching function.

Depending on the sign indices of $F_{1}$ and $F_{i}$, the $i$ th branching function $\omega_{i}$ is a monotonic function that satisfies the following:

(i) if $F_{i}^{\prime} F_{1}^{\prime}>0$, then $\omega_{i}^{\prime}(x)>0$ for almost all $x \in J_{1}$ with $\omega_{i}\left(a_{0}\right)=a_{i-1}$ and $\omega_{i}\left(a_{1}\right)=a_{i}$;

(ii) if $F_{i}^{\prime} F_{1}^{\prime}<0$, then $\omega_{i}^{\prime}(x)<0$ for almost all $x \in J_{1}$ with $\omega_{i}\left(a_{0}\right)=a_{i}$ and $\omega_{i}\left(a_{1}\right)=a_{i-1}$.

In particular, we have $\omega_{1}=x$ and

$$
F_{1}(x)=\mu^{-1}\left(\sum_{j \in I_{+}}\left[\mu\left(\omega_{j}(x)\right)-\mu\left(a_{j-1}\right)\right]+\sum_{j \in I_{-}}\left[\mu\left(a_{j}\right)-\mu\left(\omega_{j}(x)\right)\right]\right) .
$$

Instead of working on the branching function $\omega_{i}: J_{1} \rightarrow J_{i}$, it is more convenient to manipulate some functions from $J \rightarrow J$ directly. For this purpose, we can define a special class of monotonic increasing one-to-one function on the unitinterval as

Definition 2.3. A $C^{1}$ function $g: J \rightarrow J$ is said to be a generating function if $g$ meets the following requirements:

(i) $g(0)=0$ and $g(1)=1$;

(ii) $g$ is continuous;

(iii) $g^{\prime}(x)>0$ for almost all $x \in J$. 
Let $\mathcal{G}$ be the set of generating functions. Then for a given branching function $\omega_{i}: J_{1} \rightarrow$ $J_{i}, i=1,2, \ldots, r$, there exists a unique $g_{i} \in \mathcal{G}$ such that

$$
\omega_{i}(x)= \begin{cases}a_{i-1}+\left(a_{i}-a_{i-1}\right) \cdot g_{i}\left(\frac{x}{a_{1}}\right), & \text { if } F_{i}^{\prime} F_{1}^{\prime}>0, \\ a_{i}-\left(a_{i}-a_{i-1}\right) \cdot g_{i}\left(\frac{x}{a_{1}}\right), & \text { if } F_{i}^{\prime} F_{1}^{\prime}<0 .\end{cases}
$$

In particular, for $g_{1}(x)=x$, we have $\omega_{1}(x)=x$.

Therefore, for a complete chaotic map $F$, there exists a unique set of generating functions $G=\left\{g_{i}\right\}_{i=1}^{r}$, where $g_{1}=x$ and $g_{i} \in \mathcal{G}$, for $i \neq 1$. Utilizing the generating functions (which all share a common domain $J$ ) overcomes the difficulty in specifying branching functions as in (2.5) (which map $J_{1}$ to other subintervals) and hence has advantage in simplifying the general formulation. It has been shown by current author in [10] that the following.

Corollary 2.4. A complete chaotic map $F=\left\{F_{i}\right\}_{i=1}^{r}$ preserves a normalized absolutely continuous invariant measure $\mu$ if and only if there exists a set of generating functions $G=\left\{g_{i}\right\}_{i=1}^{r}$, with $g_{1}(x)=x$ and $g_{i} \in \mathcal{G}$, for $i=2,3, \ldots, r$, so that each and every branch of $F$ can be expressed as

$$
F_{i}(x)= \begin{cases}\mu^{-1}\left(\sum_{j=1}^{r} \beta_{j} \cdot g_{j}\left(g_{i}^{-1}\left(\frac{\mu(x)-\alpha_{i-1}}{\beta_{i}}\right)\right)\right), & \text { if } F_{1}^{\prime}>0, F_{i}^{\prime}>0, \\ \mu^{-1}\left(\sum_{j=1}^{r} \beta_{j} \cdot g_{j}\left(g_{i}^{-1}\left(\frac{\alpha_{i}-\mu(x)}{\beta_{i}}\right)\right)\right), & \text { if } F_{1}^{\prime}>0, F_{i}^{\prime}<0, \\ \mu^{-1}\left(1-\sum_{j=1}^{r} \beta_{j} \cdot g_{j}\left(g_{i}^{-1}\left(\frac{\alpha_{i}-\mu(x)}{\beta_{i}}\right)\right)\right), & \text { if } F_{1}^{\prime}<0, F_{i}^{\prime}>0, \\ \mu^{-1}\left(1-\sum_{j=1}^{r} \beta_{j} \cdot g_{j}\left(g_{i}^{-1}\left(\frac{\mu(x)-\alpha_{i-1}}{\beta_{i}}\right)\right)\right), & \text { if } F_{1}^{\prime}<0, F_{i}^{\prime}<0,\end{cases}
$$

where $\alpha_{i} \doteq \mu\left(a_{i}\right)$ and $\beta_{i} \doteq \alpha_{i}-\alpha_{i-1}$, for $i=1,2, \ldots, r$.

Example 2.5. Upward and downward maps.

A complete map is called upward map if $s_{i}>0$ for all $i$. We denote it as $F^{(u)}=\left\{F_{i}^{(u)}\right\}_{i=1}^{r}$. Then (2.7) is simplified to

$$
F_{i}^{(u)}(x)=\mu^{-1}\left(\sum_{j=1}^{r} \beta_{j} \cdot g_{j}\left(g_{i}^{-1}\left(\frac{\mu(x)-\alpha_{i-1}}{\beta_{i}}\right)\right)\right), \quad i=1,2, \ldots, r .
$$


A complete map is called downward map if $s_{i}<0$ for all $i$. We denote it as $F^{(d)}=$ $\left\{F_{i}^{(d)}\right\}_{i=1}^{r}$. Then (2.7) is simplified to

$$
F_{i}^{(d)}(x)=\mu^{-1}\left(1-\sum_{j=1}^{r} \beta_{j} \cdot g_{j}\left(g_{i}^{-1}\left(\frac{\mu(x)-\alpha_{i-1}}{\beta_{i}}\right)\right)\right), \quad i=1,2, \ldots, r
$$

\section{Implication Relationship}

According to (2.7), the mapping at each section, $F_{i}, i=2,3, \ldots, r$, depends on the sign index of the first branch only. Therefore, if the sign index of $k$ th $(k>1)$ branch is changed from $s_{k}$ to $s_{k}^{\prime}$, with formulation (2.7), only $k$ th branch $F_{k}$ needs to be changed. This observation reveals an important implication relationship among the set of complete maps that not only preserve an identical invariant measures but also share some analytical similarities.

Theorem 3.1. Let $F=\left\{F_{1}, \ldots, F_{k}, \ldots, F_{r}\right\}$ and $\widetilde{F}=\left\{F_{1}, \ldots, \widetilde{F}_{k}, \ldots, F_{r}\right\}$ be two complete maps defined on an identical partition $A_{F}=\left\{a_{i}\right\}_{i=0}^{r}$ of $J$ with the sign patterns differing only in kth branch (that is, $s_{k}=-\widetilde{s}_{k}$ ). Then $F$ and $\widetilde{F}$ preserve an identical invariant measure $\mu$ if and only if

$$
\widetilde{F}_{k}(x)=F_{k}\left(\varpi_{k}(x)\right)
$$

where

$$
\varpi_{k}(x)=\mu^{-1}\left(\mu\left(a_{k}\right)+\mu\left(a_{k-1}\right)-\mu(x)\right),
$$

is referred to as kth branch implication function.

Proof. Without loss of generality, we assume first $k \neq 1$.

The "if" part follows directly from Corollary 2.4, which suggests that, two maps $F$ and $\widetilde{F}$, which have identical functional forms for all branches other than $k$ th one $(k>1)$, can be generated with a same set of generating functions $\left\{g_{i}\right\}_{i=1}^{r}$. Consider the case in which $s_{1}=\widetilde{s}_{1}>0, s_{k}>0$ and $\widetilde{s}_{k}<0$, the different functional forms resulted from

$$
\begin{aligned}
& F_{k}(x)=\mu^{-1}\left(\sum_{i=1}^{r}\left(\mu\left(a_{i}\right)-\mu\left(a_{i-1}\right)\right) \cdot g_{i}\left(g_{k}^{-1}\left(\frac{\mu(x)-\mu\left(a_{k-1}\right)}{\mu\left(a_{k}\right)-\mu\left(a_{k-1}\right)}\right)\right)\right), \\
& \widetilde{F}_{k}(x)=\mu^{-1}\left(\sum_{i=1}^{r}\left(\mu\left(a_{i}\right)-\mu\left(a_{i-1}\right)\right) \cdot g_{i}\left(g_{k}^{-1}\left(\frac{\mu\left(a_{k}\right)-\mu(x)}{\mu\left(a_{k}\right)-\mu\left(a_{k-1}\right)}\right)\right)\right) .
\end{aligned}
$$

To have (3.1) hold true, the monotonicity of $g_{i}, i=1,2, \ldots, r$, implies that the following identity must be held:

$$
\frac{\mu\left(\varpi_{k}(x)\right)-\mu\left(a_{k-1}\right)}{\mu\left(a_{k}\right)-\mu\left(a_{k-1}\right)}=\frac{\mu\left(a_{k}\right)-\mu(x)}{\mu\left(a_{k}\right)-\mu\left(a_{k-1}\right)}
$$

which yields (3.2). The cases associated with $s_{1}=\widetilde{s}_{1}<0$ can be similarly verified. 
The "only if" part can be seen more straightforward from the Frobenius-perron equation (2.5).

If $s_{k}>0$ and $\widetilde{s}_{k}<0$, we must have

$$
\begin{gathered}
F_{1}(x)=\mu^{-1}\left(\sum_{i \in I_{+}-\{k\}}\left[\mu\left(\omega_{i}(x)\right)-\mu\left(a_{i-1}\right)\right]+\mu\left(\omega_{k}(x)\right)-\mu\left(a_{k-1}\right)\right. \\
\left.+\sum_{i \in I_{-}}\left[\mu\left(a_{i}\right)-\mu\left(\omega_{i}(x)\right)\right]\right), \\
\tilde{F}_{1}(x)=\mu^{-1}\left(\sum_{i \in \tilde{I}_{+}}\left[\mu\left(\tilde{\omega}_{i}(x)\right)-\mu\left(a_{i-1}\right)\right]+\mu\left(a_{k}\right)-\mu\left(\tilde{\omega}_{k}(x)\right)\right. \\
\left.+\sum_{i \in \tilde{I}_{-}-\{k\}}\left[\mu\left(a_{i}\right)-\mu\left(\tilde{\omega}_{i}(x)\right)\right]\right) .
\end{gathered}
$$

The assumption that $F_{i}(x)=\widetilde{F}_{i}(x)$ for all $i \neq k$ implies that $\omega_{i}=\widetilde{\omega}_{i}$ for all $i \neq k$ and that

$$
I_{+}-\{k\}=\tilde{I}_{+}, \quad I_{-}=\tilde{I}_{-}-\{k\}
$$

which in turn suggest that

$$
\mu\left(\omega_{k}(x)\right)-\mu\left(a_{k-1}\right)=\mu\left(a_{k}\right)-\mu\left(\tilde{\omega}_{k}(x)\right)
$$

or, equivalently,

$$
\tilde{\omega}_{k}(x)=\mu^{-1}\left(\mu\left(a_{k}\right)+\mu\left(a_{k-1}\right)-\mu\left(\omega_{k}(x)\right)\right) .
$$

Therefore, we have

$$
\tilde{\omega}_{k}^{-1}(x)=\omega_{k}^{-1}\left(\mu^{-1}\left(\mu\left(a_{k}\right)+\mu\left(a_{k-1}\right)-\mu(x)\right)\right)
$$

The facts of $F_{k}(x)=F_{1}\left(\omega_{k}^{-1}(x)\right)$ and $\widetilde{F}_{k}(x)=F_{1}\left(\tilde{\omega}_{k}^{-1}(x)\right)$, together with (3.9), thus imply (3.2) in order for the implication relationship (3.1) to hold.

The case in which $k=1$ can be proved with the same principle.

Remark 3.2. Due to the fact that $\varpi_{k}: J_{k} \rightarrow J_{k}$ is a monotonically decreasing diagonalsymmetric function in the sense of

$$
\varpi_{k}(x)=\varpi_{k}^{-1}(x)
$$


we also have

$$
F_{k}(x)=\widetilde{F}_{k}\left(\varpi_{k}(x)\right)
$$

which suggests that $F_{k}$ and $\widetilde{F}_{k}$ are mutually implied with a common implication function $\varpi_{k}$.

Definition 3.3 (implication relation). Two complete chaotic maps, $F=\left\{F_{i}\right\}_{i=1}^{r}$ and $\widetilde{F}=\left\{\widetilde{F}_{i}\right\}_{i=1}^{r}$ defined on an identical partition $A_{F}=\left\{a_{i}\right\}_{i=0}^{r}$ of $J$, are said to be mutually implied to each other if there exists a subset of indices $I^{m} \doteq\left\{i_{1}, i_{2}, \ldots, i_{m}\right\}$, with $1 \leq m \leq r$ and $i_{k} \in\{1,2, \ldots, r\}$ for $k=1,2, \ldots, m$, so that

$$
F_{k}(x)= \begin{cases}\tilde{F}_{k}\left(\varpi_{k}(x)\right), & k \in I^{m}, \\ \tilde{F}_{k}(x), & k \notin I^{m},\end{cases}
$$

where $\varpi_{k}$ is the $k$ th branch implication function defined in (3.2).

Figure 2 demonstrates the mutual-implication family (mutually implication relation was first revealed in ([9]) for two branches complete chaotic maps).

By repeatedly applying Theorem 3.1, we arrive at the following

Theorem 3.4. Two mutually implied complete chaotic maps preserve an identical invariant measure.

An important characteristic of the implication relationship is that it is invariant with smooth conjugations; that is, if $F_{a}$ and $F_{b}$ are two complete maps mutually implied by a subset of implication functions $\varpi_{k}$, for all $k \in I^{m}$, then for any one-to-one piecewise $C^{1}$ function $h, \widehat{F}_{a} \doteq h^{-1} \circ F_{a} \circ h$, and $\widehat{F}_{b} \doteq h^{-1} \circ F_{b} \circ h$ are mutually implied by $\widehat{\varpi}_{k}, k \in I^{m}$, where $\widehat{\varpi}_{k} \doteq h^{-1}\left(\varpi_{k}(h(x))\right)$, for all $k \in I^{m}$. An application of mutual implication relationship in constructing an opposite for a two-segmental complete map is offered in [9].

The implication relationship (the set of implication functions) is uniquely determined by the partition $A_{F}=\left\{a_{i}\right\}_{i=0}^{r}$ and the invariant measure preserved $\mu$, but is independent of the generating functions $G$. For any complete map $F=\left\{F_{i}\right\}_{i=1}^{r}$ preserving an invariant measure $\mu$, there exists a unique implied family of $F$, denoted as $\Psi(F)$, in which each and every map can be derived from the other through replacing some branches with their implied counterparts. On the other hand, for each and every sign pattern $S=\left\{s_{i}\right\}_{i=1}^{r}$, there associates one and only one map in an implied family. The size of this family is thus always $2^{r}$. Moreover, for any $\theta=\left\{\theta_{i}\right\}_{i=1}^{r} \in \Psi(F)$, either $\theta_{i}=F_{i}$ or $\theta_{i}=F_{i} \circ \varpi_{i}$, for $i=1,2, \ldots, r$. And hence, the family $\Psi(F)$ is uniquely specified by $2 r$ mappings and will be denoted as $\Psi(F)=\left[F_{i}^{u}, F_{i}^{d}\right]_{i=1}^{r}$, or,

$$
\Psi(F)=\left[\begin{array}{cc}
F_{1}^{u}, & F_{1}^{u} \circ \varpi_{1} \\
\vdots & \vdots \\
F_{i}^{u}, & F_{i}^{u} \circ \varpi_{i} \\
\vdots & \vdots \\
F_{r}^{u}, & F_{r}^{u} \circ \varpi_{r}
\end{array}\right]=\left[\begin{array}{cc}
F_{1}^{d} \circ \varpi_{1}, & F_{1}^{d} \\
\vdots & \vdots \\
F_{i}^{d} \circ \varpi_{i}, & F_{i}^{d} \\
\vdots & \vdots \\
F_{r}^{d} \circ \varpi_{r}, & F_{r}^{d}
\end{array}\right] .
$$



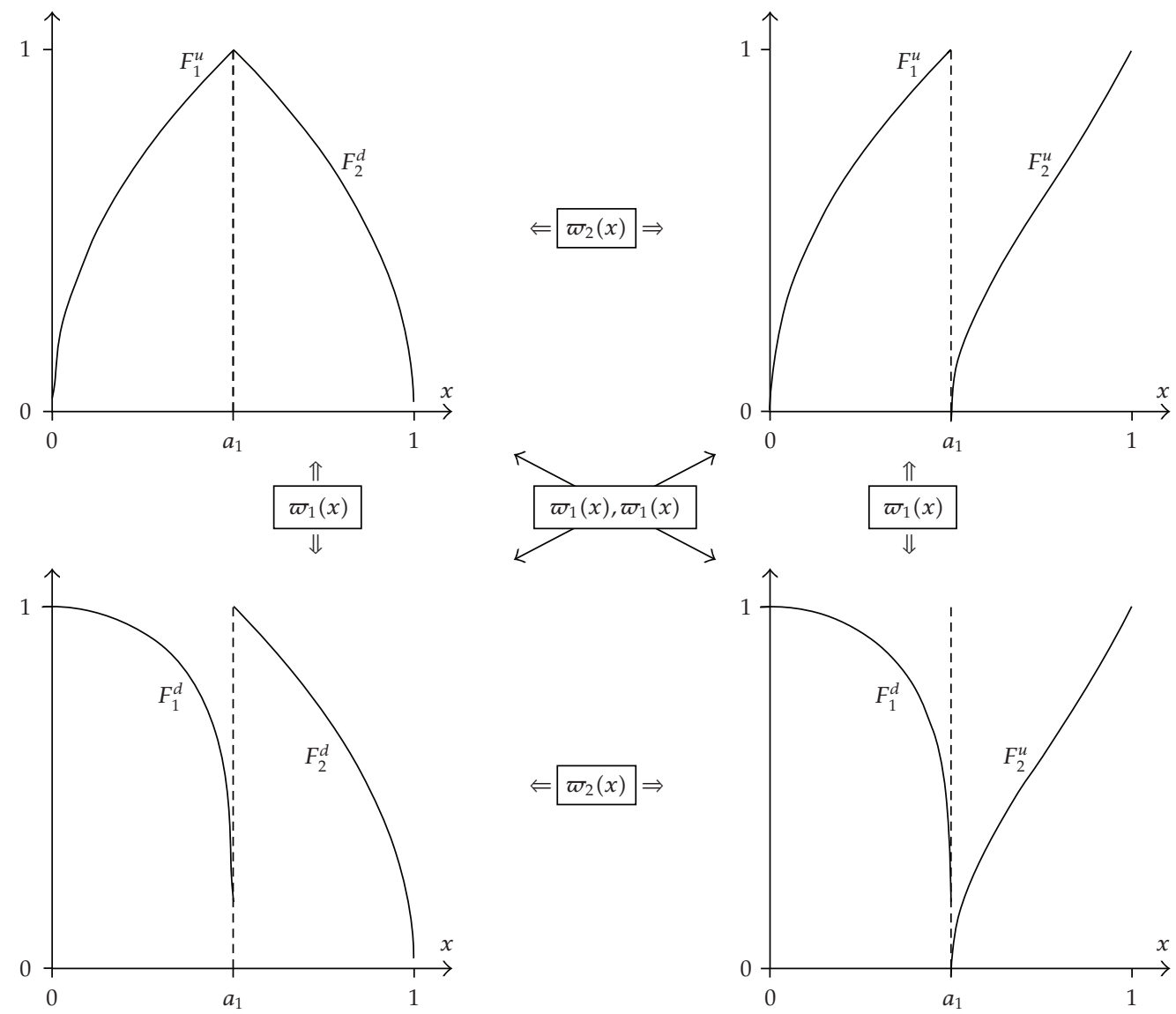

Figure 2: Mutually implied family.

Due to the uniqueness of implied family, just substituting (3.2) into (2.8) yields the following.

Theorem 3.5. A complete map $F=\left\{F_{i}\right\}_{i=1}^{r}$ preserves a normalized absolutely continuous invariant measure $\mu$ if and only if there exists a set of generating functions $G=\left\{g_{i}\right\}_{i=1}^{r}$, with $g_{1}(x)=x$ and $g_{i} \in \mathcal{G}$, for $i=2,3, \ldots, r$, so that each and every branch of $F$ can be expressed as

$$
F_{i}(x)=\left\{\begin{array}{ll}
\mu^{-1}\left(\sum_{j=1}^{r} \beta_{j} \cdot g_{j}\left(g_{i}^{-1}\left(\frac{\mu(x)-\alpha_{i-1}}{\beta_{i}}\right)\right)\right), & \text { if } F_{i}^{\prime}>0, \\
\mu^{-1}\left(\sum_{j=1}^{r} \beta_{j} \cdot g_{j}\left(g_{i}^{-1}\left(\frac{\alpha_{i}-\mu(x)}{\beta_{i}}\right)\right)\right), & \text { if } F_{i}^{\prime}<0,
\end{array} \quad i=1,2, \ldots, r,\right.
$$

where $\alpha_{i} \doteq \mu\left(a_{i}\right)$ and $\beta_{i} \doteq \alpha_{i}-\alpha_{i-1}$, for $i=1,2, \ldots, r$. 
We further show that any member of an implication family shares the same degree of chaos.

Theorem 3.6. All members of an implication family $\Psi(F)$ have an identical Lyapunov exponent.

Proof. Due to the facts that two conjugated complete maps have an identical Lyapunov exponent and that the implication relationship is invariant of topological conjugation, we can proceed with the complete maps that preserve the uniform invariant density $(\mu(x) \equiv x)$.

For a complete map $F$ defined on a partition $A_{F}=\left\{a_{i}\right\}_{i=0}^{r}$ of $J$, its Lyapunov exponent, denoted as $\Lambda(F)$, is given by $\Lambda(F)=\sum_{i=1}^{r} \lambda_{i}\left(F_{i}\right)$, where

$$
\lambda_{i}\left(F_{i}\right) \doteq \int_{a_{i-1}}^{a_{i}} \ln \left(\left|F_{i}^{\prime}(x)\right|\right) \varphi(x) d x, \quad \forall i \in\{1,2, \ldots, r\} .
$$

It is thus sufficient to prove that the identity $\lambda_{i}\left(F_{i}\right)=\lambda_{i}\left(\widetilde{F}_{i}\right)$ holds true for all $i \in$ $\{1,2, \ldots, r\}$, where $\widetilde{F}_{i}=F_{i}\left(\varpi_{i}\right)$.

Indeed, since $\mu(x) \equiv x$ (and hence $\varphi(x)=1$ ), we have $\varpi_{i}(x)=a_{i}+a_{i-1}-x$ so that

$$
\lambda_{i}\left(F_{i}\right)=\int_{a_{i-1}}^{a_{i}} \ln \left(\left|F_{i}^{\prime}\left(\varpi_{i}\right) \varpi_{i}^{\prime}\right|\right) d x=\int_{a_{i-1}}^{a_{i}} \ln \left(\left|F_{i}^{\prime}\left(a_{i}+a_{i-1}-x\right)\right|\right) d x=\lambda_{i}\left(F_{i}\right),
$$

which completes the proof.

For $g_{i}(x)=x, i=1,2, \ldots, r,(3.14)$ simplifies to

$$
F_{i}(x)=\left\{\begin{array}{ll}
\mu^{-1}\left(\frac{\mu(x)-\alpha_{i-1}}{\beta_{i}}\right), & \text { if } F_{i}^{\prime}>0, \\
\mu^{-1}\left(\frac{\alpha_{i}-\mu(x)}{\beta_{i}}\right), & \text { if } F_{i}^{\prime}<0,
\end{array} \quad i=1,2, \ldots, r,\right.
$$

which is nothing but a topological conjugate of complete piecewise linear chaotic map defined by

$$
T_{i}(x)=\left\{\begin{array}{ll}
\frac{x-\alpha_{i-1}}{\alpha_{i}-\alpha_{i-1}}, & \text { if } T_{i}^{\prime}>0, \\
\frac{\alpha_{i}-x}{\alpha_{i}-\alpha_{i-1}}, & \text { if } T_{i}^{\prime}<0,
\end{array} \quad i=1,2, \ldots, r\right.
$$

with $A_{T}=\left\{\alpha_{i}\right\}_{i=1}^{r}$.

It is well known that $T=\left\{T_{i}\right\}_{i=1}^{r}$ preserves the Lebesgue measure. This observation reveals the fact that the class of complete chaotic maps that are conjugated to the complete piecewise linear chaotic maps consists of only a subset of all complete chaotic maps that preserve the same absolute continuous invariant measures.

For the special case in which $g_{i}(x)=x$ for all $i$, it is immediately observed that

$$
\lambda(F)=-\sum_{i=1}^{r} \beta_{i} \ln \beta_{i} .
$$


Finally, we just point out that (3.14) is not the unique way to constructing complete chaotic maps with given $\mu, A_{F}$, and G. For instance, we may substitute (3.2) into (2.9) alternatively so as to obtain another implied family given by $\widehat{F}=\left\{\widehat{F}_{i}\right\}_{i=1}^{r}$, where

$$
\widehat{F}_{i}(x)=\left\{\begin{array}{ll}
\mu^{-1}\left(1-\sum_{j=1}^{r} \beta_{j} \cdot g_{j}\left(g_{i}^{-1}\left(\frac{\alpha_{i}-\mu(x)}{\beta_{i}}\right)\right)\right), & \text { if } F_{i}^{\prime}>0, \\
\mu^{-1}\left(1-\sum_{j=1}^{r} \beta_{j} \cdot g_{j}\left(g_{i}^{-1}\left(\frac{\mu(x)-\alpha_{i-1}}{\beta_{i}}\right)\right)\right), & \text { if } F_{i}^{\prime}<0,
\end{array} \quad i=1, \ldots, r .\right.
$$

$\widehat{F}$ defined in (3.20) is identical to $F$ defined in (3.14) if and only if $g_{i}(x)=x$ for all $i$. However, it is easy to see that $\widehat{F}_{i}$ is nothing but to implement formulation (3.14) with a set of new generating functions $\widehat{G}=\left\{\widehat{g}_{i}\right\}_{i=1}^{r}$ with $\widehat{g}_{i}(x)=1-g_{i}(1-x)$ for all $i$. Therefore, we will concentrate on the formulation (3.14) only. Moreover, we can further show that two families $\Psi(F)$ and $\Psi(\widehat{F})$ have an identical Lyapunov exponent.

\section{Numerical Examples}

Example 4.1. Consider a 3-to-1 complete chaotic map with $\mu(x)=2 \arcsin (\sqrt{x}) / \pi(\varphi(x)=$ $1 /(\pi \sqrt{x(1-x)})), A=\{0,1 / 4,3 / 4,1\}$, and $G=\{x, x, x\}$.

With $\alpha_{0}=0, \alpha_{1}=\mu(1 / 4)=1 / 3, \alpha_{2}=\mu(3 / 4)=2 / 3$, and $\alpha_{3}=1$, we have $\beta_{i}=\alpha_{i}-\alpha_{i-1}=$ $1 / 3$ for all $i$.

For this simple generating functions, we can apply directly formulation (3.17) to get

$$
\begin{aligned}
& F_{1}^{(u)}(x)=F_{2}^{(d)}(x)=F_{3}^{(u)}(x)=f(x) \doteq x(4 x-3)^{2}, \\
& F_{1}^{(d)}(x)=F_{2}^{(u)}(x)=F_{3}^{(d)}(x)=g(x) \doteq(1-x)(1-4 x)^{2} .
\end{aligned}
$$

Moreover, $\lambda(F)=-\sum_{i=1}^{3} \beta_{i} \ln \beta_{i}=\ln 3$.

The three branching functions are given by

$$
\begin{gathered}
\varpi_{1}(x)=\mu^{-1}\left(\alpha_{1}+\alpha_{0}-\mu(x)\right)=\frac{1}{4}+\frac{1}{2} x-\frac{1}{2} \sqrt{3 x(1-x)}, \\
\varpi_{2}(x)=\mu^{-1}\left(\alpha_{2}+\alpha_{1}-\mu(x)\right)=1-x \\
\varpi_{3}(x)=\mu^{-1}\left(\alpha_{3}+\alpha_{2}-\mu(x)\right)=\frac{1}{4}+\frac{1}{2} x+\frac{1}{2} \sqrt{3 x(1-x)} .
\end{gathered}
$$




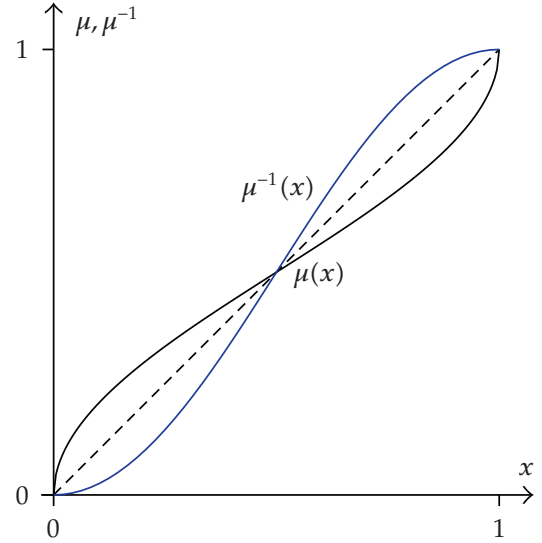

(a) Invariant measure $\mu$ and its inverse

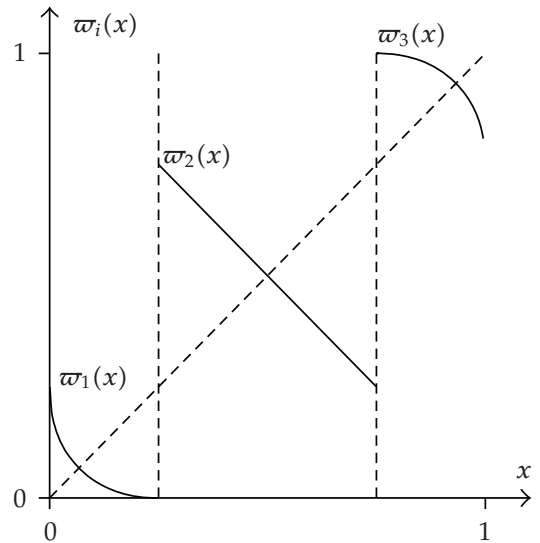

(b) Branching functions

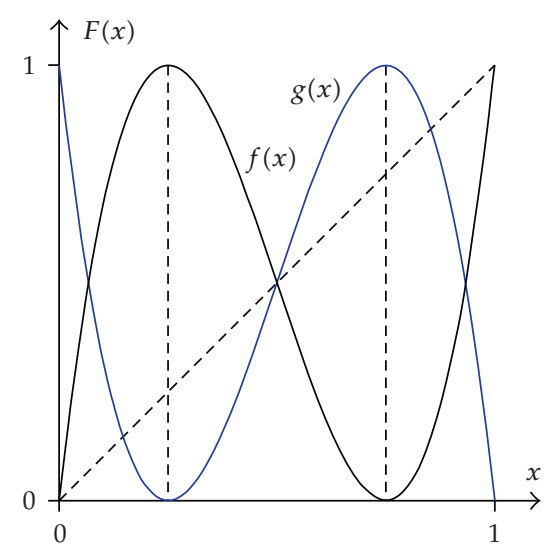

(c) $F$ resulted from $G=\{x, x, x\}$

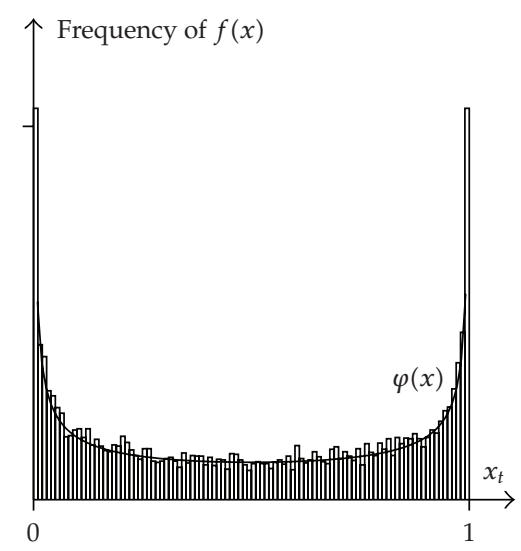

(d) $\phi(x)$ and frequency of $f(x)$

Figure 3: Illustration of Example 4.1.

It is interesting to verify that

$$
\begin{gathered}
f\left(\varpi_{i}(x)\right)=g(x), \\
g\left(\varpi_{3}(x)\right)=f(x)
\end{gathered}
$$

for $i=1,2,3$.

The above conclusions are illustrated in Figure 3. In particular, Figure 3(d) provides a typical realized frequency from $f(x)=x(4 x-3)^{2}$.

Example 4.2. Consider a 3-to-1 complete chaotic map with $\mu(x)=x^{2}(\varphi(x)=2 x)$ and $A_{F}=$ $\{0,1 / 3,2 / 3,1\}$.

With $\alpha_{0}=0, \alpha_{1}=1 / 9, \alpha_{2}=4 / 9$, and $\alpha_{3}=1$, we have $\beta_{1}=1 / 9, \beta_{2}=1 / 3$, and $\beta_{3}=5 / 9$, respectively. 
The branching functions turn out to be

$$
\begin{aligned}
& \varpi_{1}(x)=\mu^{-1}\left(\alpha_{1}+\alpha_{0}-\mu(x)\right)=\sqrt{\frac{1}{9}-x^{2}} \\
& \varpi_{2}(x)=\mu^{-1}\left(\alpha_{2}+\alpha_{1}-\mu(x)\right)=\sqrt{\frac{5}{9}-x^{2}} \\
& \varpi_{3}(x)=\mu^{-1}\left(\alpha_{3}+\alpha_{2}-\mu(x)\right)=\sqrt{\frac{13}{9}-x^{2}}
\end{aligned}
$$

(i) Consider first the set of generating functions, $G^{(1)} \doteq\{x, x, x\}$, then (3.17) yields

$$
\begin{array}{cl}
F_{1}^{(u)}(x)=3 x, & F_{1}^{(d)}(x)=F_{1}^{(u)}\left(\varpi_{1}(x)\right)=\sqrt{1-9 x^{2}}, \\
F_{2}^{(u)}(x)=\sqrt{3 x^{2}-\frac{1}{3}}, \quad F_{2}^{(d)}(x)=F_{2}^{(u)}\left(\varpi_{2}(x)\right)=\frac{1}{3} \sqrt{12-27 x^{2}}, \\
F_{3}^{(u)}(x)=\sqrt{\frac{9}{5} x^{2}-\frac{4}{5}}, \quad F_{3}^{(d)}(x)=F_{3}^{(u)}\left(\varpi_{3}(x)\right)=\frac{3}{5} \sqrt{5-5 x^{2}} .
\end{array}
$$

It is straightforward to verify that $\lambda(F) \simeq 0.93689=-\sum_{i=1}^{r} \beta_{i} \ln \beta_{i}$. yields

(ii) Alternatively, if we consider the set of generation functions $G^{(2)} \doteq\left\{x, x^{2}, x\right\}$,

$$
\begin{aligned}
\bar{F}_{1}^{(u)}(x)= & \mu^{-1}\left(\beta_{1} \cdot g_{1}\left(g_{1}^{-1}\left(\frac{\mu(x)-\alpha_{0}}{\beta_{1}}\right)\right)+\beta_{2} \cdot g_{2}\left(g_{1}^{-1}\left(\frac{\mu(x)-\alpha_{0}}{\beta_{1}}\right)\right)\right. \\
& \left.+\beta_{3} \cdot g_{3}\left(g_{1}^{-1}\left(\frac{\mu(x)-\alpha_{0}}{\beta_{1}}\right)\right)\right) \\
= & x \sqrt{6+27 x^{2}}, \\
\bar{F}_{2}^{(u)}(x)= & \mu^{-1}\left(\beta_{1} \cdot g_{1}\left(g_{2}^{-1}\left(\frac{\mu(x)-\alpha_{1}}{\beta_{2}}\right)\right)+\beta_{2} \cdot g_{2}\left(g_{2}^{-1}\left(\frac{\mu(x)-\alpha_{1}}{\beta_{2}}\right)\right)\right. \\
& \left.\quad+\beta_{3} \cdot g_{3}\left(g_{2}^{-1}\left(\frac{\mu(x)-\alpha_{1}}{\beta_{2}}\right)\right)\right) \\
= & \frac{1}{3} \sqrt{2 \sqrt{3\left(9 x^{2}-1\right)}+9 x^{2}-1,} \\
\bar{F}_{3}^{(u)}(x)= & \mu^{-1}\left(\beta_{1} \cdot g_{1}\left(g_{3}^{-1}\left(\frac{\mu(x)-\alpha_{2}}{\beta_{3}}\right)\right)+\beta_{2} \cdot g_{2}\left(g_{3}^{-1}\left(\frac{\mu(x)-\alpha_{2}}{\beta_{3}}\right)\right)\right. \\
& \left.\quad+\beta_{3} \cdot g_{3}\left(g_{3}^{-1}\left(\frac{\mu(x)-\alpha_{2}}{\beta_{3}}\right)\right)\right) \\
= & \frac{1}{5} \sqrt{\left(9 x^{2}-4\right)\left(3 x^{2}+2\right) .}
\end{aligned}
$$




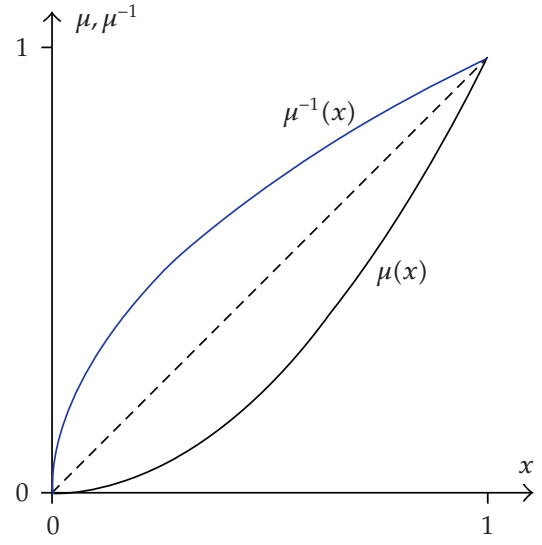

(a) Invariant measure $\mu$ and its inverse

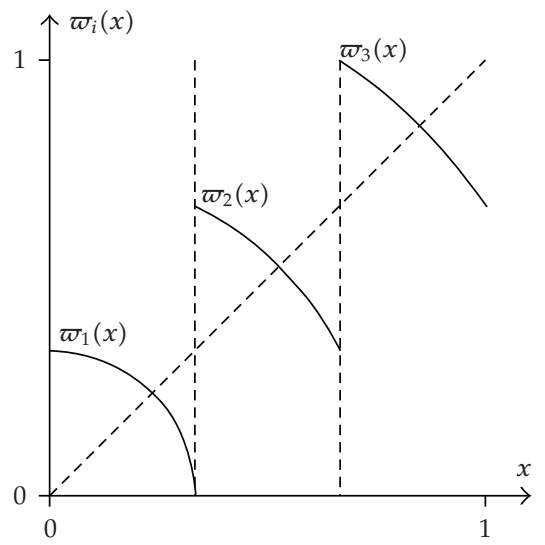

(b) Branching functions

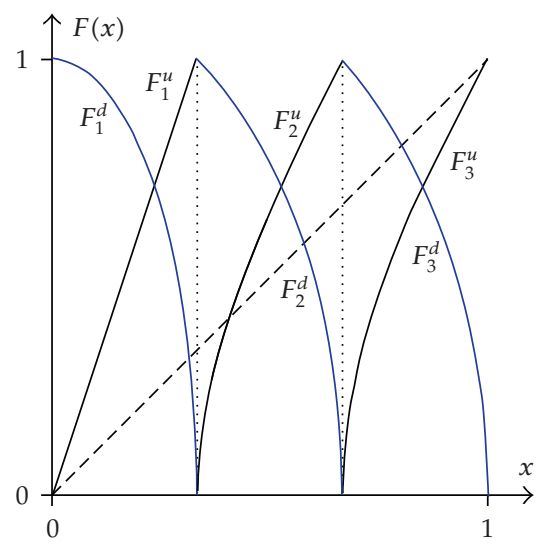

(c) $F$ resulted from $G=\{x, x, x\}$

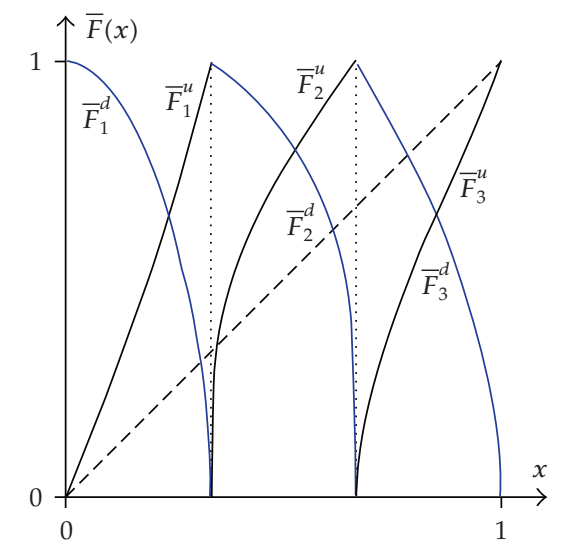

(d) $\bar{F}$ resulted from $G=\left\{x, x^{2}, x\right\}$

Figure 4: Illustration of Example 4.2.

Accordingly, we have

$$
\begin{gathered}
\bar{F}_{1}^{(d)}(x)=F_{1}^{(u)}\left(\varpi_{1}(x)\right)=\sqrt{\left(1-3 x^{2}\right)\left(1-9 x^{2}\right)}, \\
\bar{F}_{2}^{(d)}(x)=F_{2}^{(u)}\left(\varpi_{2}(x)\right)=\frac{1}{3} \sqrt{4-9 x^{2}+2 \sqrt{3\left(4-9 x^{2}\right)}}, \\
\bar{F}_{3}^{(d)}(x)=F_{3}^{(u)}\left(\varpi_{3}(x)\right)=\frac{1}{5} \sqrt{3\left(1-x^{2}\right)\left(19-9 x^{2}\right)} .
\end{gathered}
$$

Straight calculation reveals that $\lambda(F)=0.89123$.

The above conclusions are illustrated in Figure 4. 


\section{References}

[1] D. Pingel, P. Schmelcher, and F. K. Diakonos, “Theory and examples of the inverse Frobenius-Perron problem for complete chaotic maps," Chaos, vol. 9, no. 2, pp. 357-366, 1999.

[2] W. Huang, "Characterizing chaotic processes that generate uniform invariant density," Chaos, Solitons $\mathcal{E}$ Fractals, vol. 25, no. 2, pp. 449-460, 2005.

[3] W. Huang, "On the complete chaotic transformations that preserve symmetric invariant densities," Chaos, Solitons \& Fractals, vol. 38, no. 4, pp. 1065-1074, 2008.

[4] W. Huang, "On complete chaotic maps with tent-map-like structures," Chaos, Solitons E Fractals, vol. 24, no. 1, pp. 287-299, 2005.

[5] W. Huang, "Constructing complete chaotic maps with reciprocal structures," Discrete Dynamics in Nature and Society, vol. 2005, no. 3, pp. 357-372, 2005.

[6] W. Huang, "Constructing chaotic transformations with closed functional forms," Discrete Dynamics in Nature and Society, vol. 2006, Article ID 42876, 16 pages, 2006.

[7] A. Matsumoto, "Density function of piecewise linear transformation," Journal of Economic Behavior and Organization, vol. 56, no. 4, pp. 631-653, 2005.

[8] W. Huang and Y. Zhang, "Distributional dynamics of cautious economic adjustment processes," Journal of Economic Behavior and Organization, vol. 62, no. 3, pp. 389-407, 2007.

[9] W. Huang, "Constructing an opposite map to a specified chaotic map," Nonlinearity, vol. 18, no. 3, pp. 1375-1391, 2005.

[10] W. Huang, "On the complete chaotic maps that preserve prescribed absolutely continuous invariant densities," in Topics on Chaotic Systems: Selected Papers from CHAOS 2008 International Conference, C. H. Skiadas, I. Dimotikalis, and C. Skiadas, Eds., pp. 166-173, Crete, Greece, June 2008. 


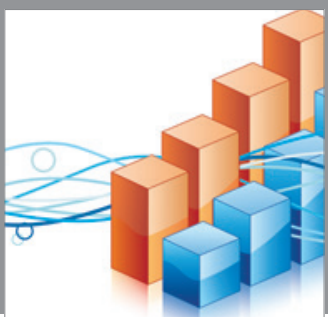

Advances in

Operations Research

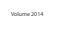

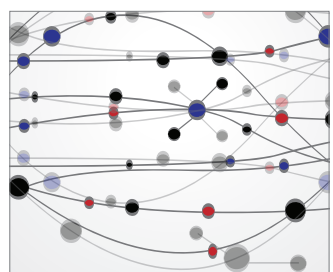

\section{The Scientific} World Journal
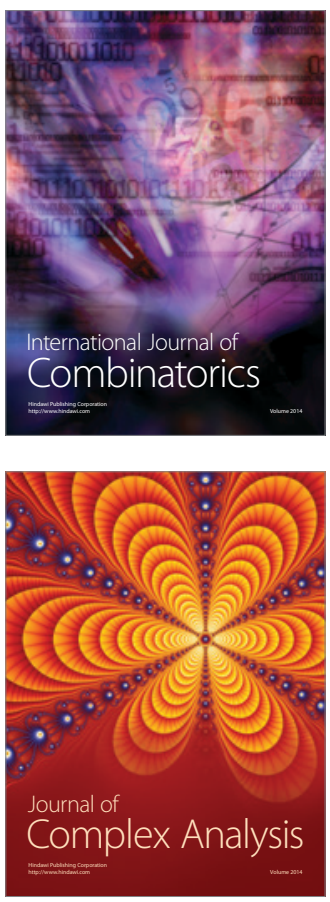

International Journal of

Mathematics and

Mathematical

Sciences
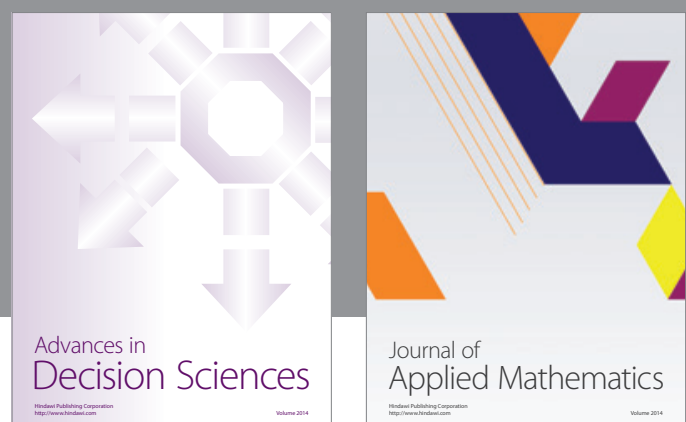

Journal of

Applied Mathematics
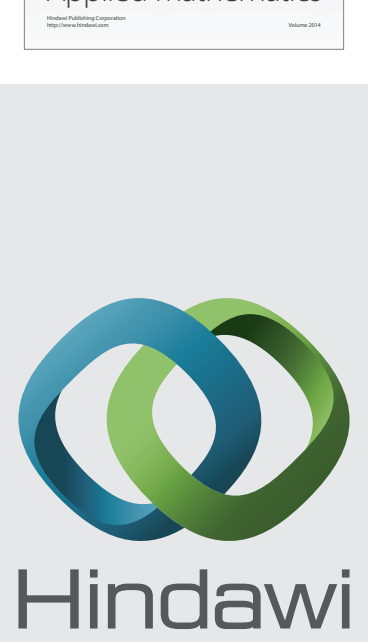

Submit your manuscripts at http://www.hindawi.com
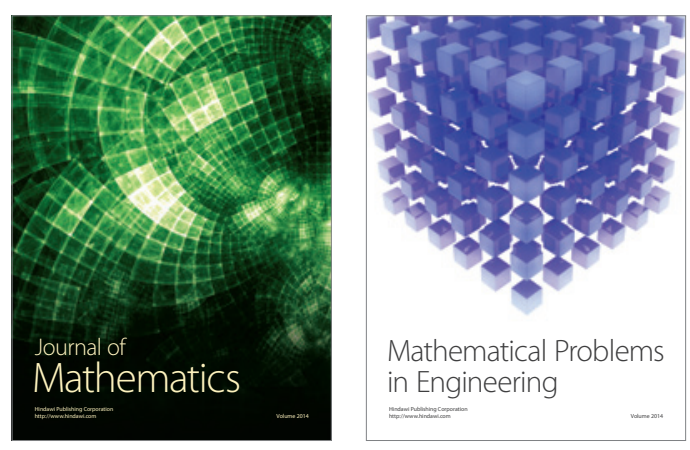

Mathematical Problems in Engineering
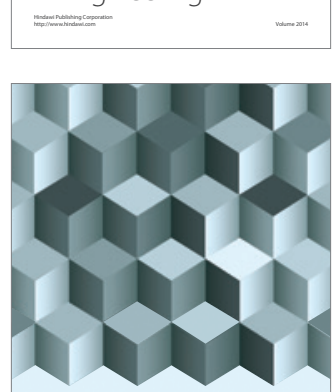

Journal of

Function Spaces
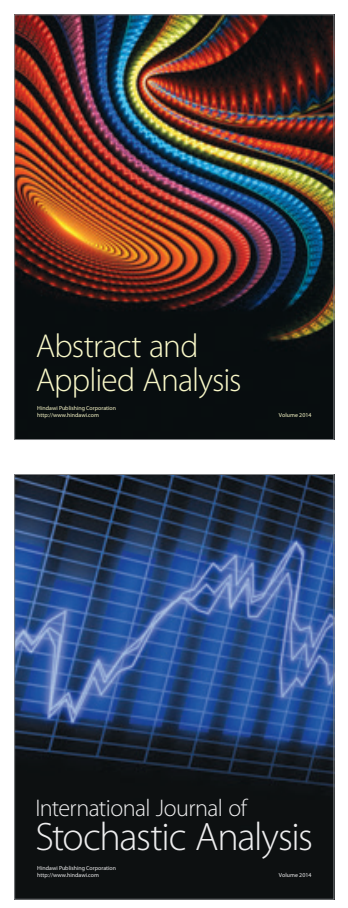

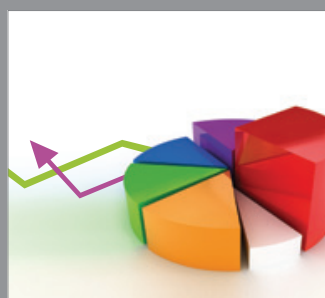

ournal of

Probability and Statistics

Promensencen
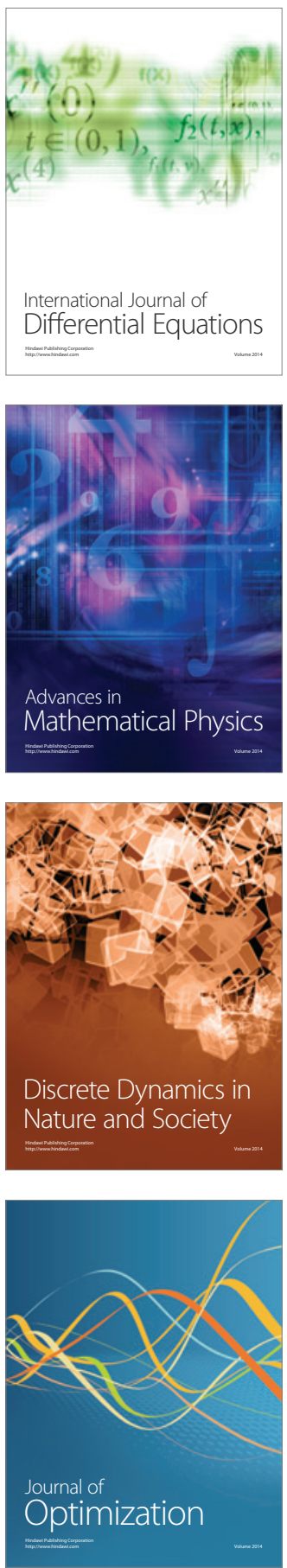